\title{
Interference filters as nonlinear decision-making elements for three-spot pattern recognition and associative memories
}

\author{
L. Wang, V. Esch, R. Feinleib, L. Zhang, R. Jin, H. M. Chou, Robert W. Sprague, H. Angus Macleod, G. \\ Khitrova, Hyatt M. Gibbs, Kelvin Wagner, and Demetri Psaltis
}

\begin{abstract}
Simple patterns consisting of three spots $(V$ and $\Gamma)$ have been recognized by dividing, shifting, and recombining beams onto bistable $\mathrm{ZnS}$ interference filters. This experiment demonstrates AND-gate operation, cascading, and a moderate amount of parallelism, but a laser power of several watts was required and the response times were several milliseconds. An associative memory for fingerprint identification has been constructed using a VanderLugt correlator and an interference filter as a reflective thresholding device.
\end{abstract}

\section{Introduction}

The two all-optical systems described here demonstrate the use of nonlinear optical devices to make decisions. The devices are demonstrated in two different types of system. In the three-spot recognition system multiple nonlinear elements are necessary for a pattern recognition. Conventional optical components are employed to manipulate the orientation of the processing beams. In the associative memory system, conventional optical techniques play the key processing role through matched filtering of a partial input. The nonlinear optical decision-making element utilizes the output of the matched filter, the crosscorrelation beams, to decide closest match. The corresponding hologram is then read out with a counterpropagating beam.

\section{Three-Spot Pattern Recognition}

\section{A. Goal and Motivation}

The goal of the experiment was to identify the locations of simple patterns consisting of three beams (spots), for example, the $V$ and $\Gamma$ defined in Fig. 1, in an input array. In this case a $2 \times 9$ beam array was used,

Demetri Psaltis is with California Institute of Technology, Pasadena, California 91125; the other authors are with University of Arizona, Optical Sciences Center, Tucson, Arizona 85721.

Received 28 January 1988.

0003-6935/88/091715-06\$02.00/0.

(C) 1988 Optical Society of America. with several randomly selected beams blocked. Such a three-spot pattern could be recognized using a single interference filter as a three-input AND gate, but here two successive two-input AND-gate operations are performed to emphasize the capability of cascading the nonlinear interference filters. ${ }^{1,2}$

One-bit addition by the technique of symbolic substitution ${ }^{3,4}$ has been demonstrated previously using interference filters, showing that very simple patterns can be useful in digital optical computing. ${ }^{5}$ The present experiment shows more parallelism and the potential for nonlinear decision-making in optical pattern recognition. Furthermore it suggests that the best use of nonlinear decision-making may not be for numerical computing, already done so well by electronics. Rather the best use may be to combine more traditional optical techniques of image processing, for example, real-time correlations, with a limited amount of nonlinear decision-making. Only if several correlation peaks or other events occur sufficiently simultaneously will the system activate an appropriate response. The present experiment is admittedly slow and power hungry, but it illustrates the ability to recognize the simultaneous occurrence of three bright spots with all-optical decision-making.

\section{B. Experimental Apparatus}

The apparatus is diagrammed in Fig. 1. A Coherent Innova-20 cw argon laser operating at $514 \mathrm{~nm}$ was the light source. The beam was modulated by a rotating halfwave plate and polarization beam splitter, with a maximum transmission of $\simeq 76 \%$ and minimum of $3 \%$. Two binary phase gratings, that produce two and nine approximately equal beams, respectively, were orient- 


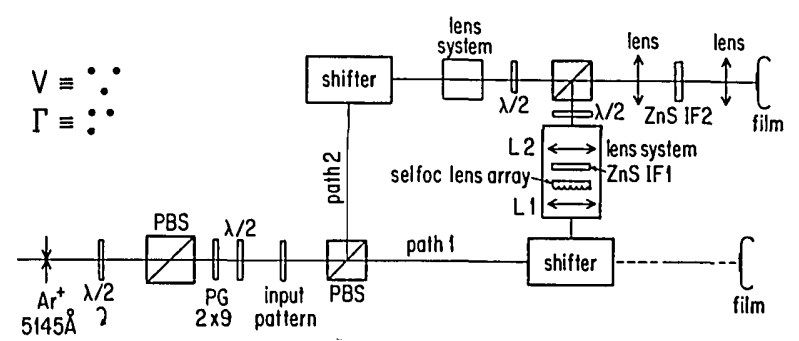

Fig. 1. Experimental arrangement for digital pattern recognition.

ed orthogonally and used in tandem to divide the 2.3$\mathrm{W}$ input beam into $2 \times 9$ beams. Patterns were then encoded into the beam array by blocking selected beams. The power in each beam was $58 \mathrm{~mW}$ within $10 \%$, after reducing the two central order beams by $\sim 30 \%$.

$\mathrm{ZnS}$ interference filters grown in the Thin Film Coating Laboratory at the University of Arizona were used as nonlinear decision-making devices. The peak linear transmissions were $54 \%$ and $40 \%$ and the full widths were 17 and $13 \AA$, respectively. With the appropriate scaling of the input intensities they require two or more inputs to switch to the high transmission state, therefore these nonlinear devices can be used as bistable AND gates. The devices have millisecond recovery times; they operate in the visible, facilitating alignment; and they are relatively easy to grow or inexpensive to buy. These characteristics make them attractive for demonstrating optical computing concepts. However, their lack of uniformity and longterm stability make it necessary to search for good regions repeatedly. The minimum switch-up powers were 9 and $16 \mathrm{~mW}$, respectively, in a $100-\mu \mathrm{m}$ diam spot. In multiple-beam experiments the focusing was often imperfect, so that typical switch-up powers were 20-40 $\mathrm{mW}$ on the filter and the nonlinear transmission was typically $10-25 \%$. However, the contrast was $\simeq 3.5$ (ratio between the on and off state transmission), permitting fan-out and cascading. Other system losses limited the number of AND-gate operations per watt to 5 or 10 , thereby limiting the parallelism achievable. The filters were grown for near-perpendicular operation at $514.5 \mathrm{~nm}$ minimizing the complications of parallel operation on a tilted device.

Patterns were tested by sequentially identifying portions of the pattern. This operation was performed in conjunction with the shifters. The array of beams was first split into two arrays. One of the arrays was then shifted and recombined with the unshifted array on the interference filter. In this way a portion of the pattern was tested. For example, a diagonal shift was needed to identify the left portion of a $V$. Either a Mach-Zehnder interferometer or triangularinterferometer setup could be used as a shifter.

Each lens system in Fig. 1 focuses each of the input beams so that the parallel logic operations could be performed. The spots on the filters were $\sim 75 \mu \mathrm{m}$ in diameter and $1 \mathrm{~mm}$ apart. On IF1 the shifted input pattern and original pattern were superimposed by the

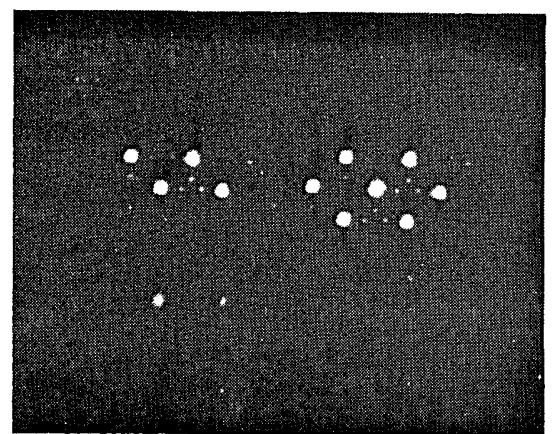

Fig. 2. Recognition of $V$. Input pattern is in the upper left. Shifted pattern is in the upper right. The result is in the lower left, showing the occurrence of the right side of a $V$ in only one location.

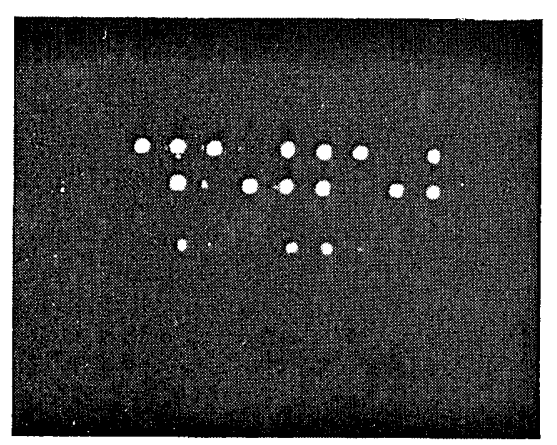

Fig. 3. Recognition of $\Gamma$. Input pattern is the upper two rows. The output of the two successive AND-gate operations used to look for $\Gamma s$ is shown underneath, clearly indicating the presence of three $\Gamma$ s.

shifter and focused by the lens system. On IF2 the pattern, approximately shifted to search for the third spot, was focused on the reimaged output of IF1. The lens systems consisted of two lenses, L1 and L2, and a Selfoc lens array. The combination of $\mathrm{L} 1$ and the Selfoc lens array was used to provide enough intensity for AND-gate operation in IF1. Lens L2 acted as a relay lens to pass signals to the next stage IF2. To minimize aberrations in the system, the lens system in path 2 was symmetrical to path 1, except that no logic operation was needed in this path for recognizing the third spot.

\section{Experimental Results}

The first step in recognizing a $V$ is shown in Fig. 2. The upper left shows the input pattern, encoded in a 2 $\times 4$ format. The unshifted pattern and the pattern shifted diagonally down and to the left are superimposed in the upper right. The output of the AND-gate operation, shown below the input, gives a bright spot at the bottom of the $V$. Of course, this only shows that the right part of the $V$ is present. A second AND operation, with the pattern shifted diagonally down to the right, completes the $V$ recognition.

Recognition of three $\Gamma$ s in a $2 \times 9$ input pattern is shown in Fig. 3. Clearly any three-spot pattern or simultaneous occurrences could be used to trigger an output response from this all-optical coincidence circuit. 


\section{Associative Memory}

\section{A. Motivation and Concept}

The $V$ and $\Gamma$ experiments illustrate AND-gate operation, cascading, and a moderate amount of parallelism. However they also illustrate the difficulties of dividing a beam into many beams so that each decision-making pixel receives the same amount of power in approximately the same cross-sectional area. These geometrical optics difficulties and the high power requirements of large arrays of nonlinear decision-making pixels are formidable hurdles to massively parallel nonlinear optical computing systems. This suggests the utilization of more conventional techniques of image processing such as correlation with a limited amount of nonlinear decision-making.

Recent work has dealt with associative memories ${ }^{6,7}$ based on various thresholding and feedback schemes, and several optical implementations have been demonstrated $^{8-11}$ (a good general reference for this work is the 1 Dec. 1987 issue of Applied Optics on Neural Networks.) The optical associative memory discussed here, similar to that presented by Soffer et al., ${ }^{12} \mathrm{de}-$ cides which stored object most closely matches a partial-object input. The associative memory employs two elements. The first element, a VanderLugt correlator, gives an analog degree-of-match value, and the second, an optically bistable interference filter, gives a binary thresholding response.

The idealized system works by writing Fourier transform phase holograms, sequentially multiplexed in reference beam angle, of the different objects. The test object is then correlated with the stored objects giving angularly separated cross-correlation beams. Each beam is focused onto an optically bistable interference filter where the decision of closest match is made at the central peak positions.

The interference filter was used in conjunction with a holding beam in this experiment. Power is supplied, in this case exactly counterpropagating with each correlation beam, to bias the device very close to the switching threshold. Only the minimum switching power is then necessary to push the device into the transmissive mode. This reduces the required correlation power, and thus the power through the hologram. The counterpropagating bias beam also facilitates the recall of stored objects.

One of the beams, corresponding to the most closely matched stored object, is sufficiently intense to provide the minimum switching power, allowing the holding beam to collinearly counterpropagate to the hologram. Because it is a plane wave at the proper incident angle to the hologram it reads out the appropriate object from the hologram, and the object is propagated back through the optical system. Also, because the hologram is thick, only this one object is Bragg matched; no other subjects are read out. This is the autoassociative mode of operation, where a partial object input recalls a reconstruction of the same object.

If the holding beam is propagated to another hologram or transparency, a heteroassociative recall can

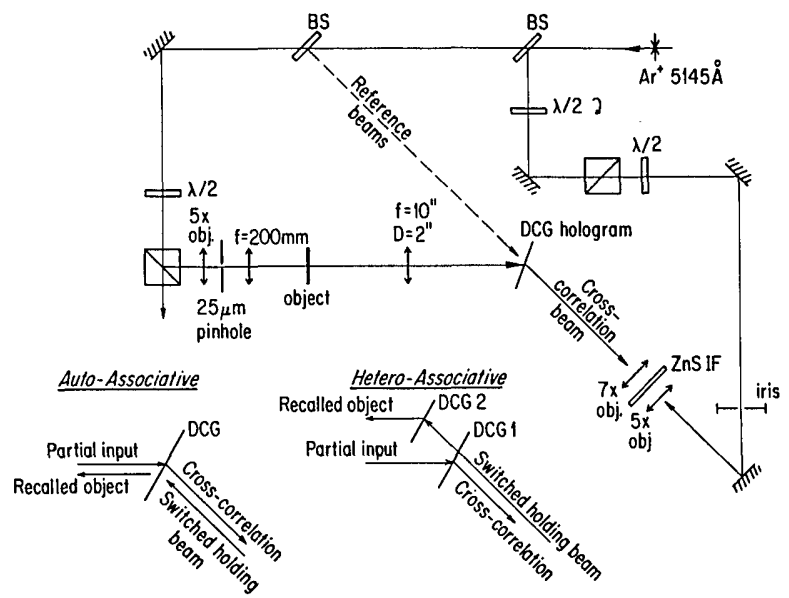

Fig. 4. Experimental arrangement for associative memory.
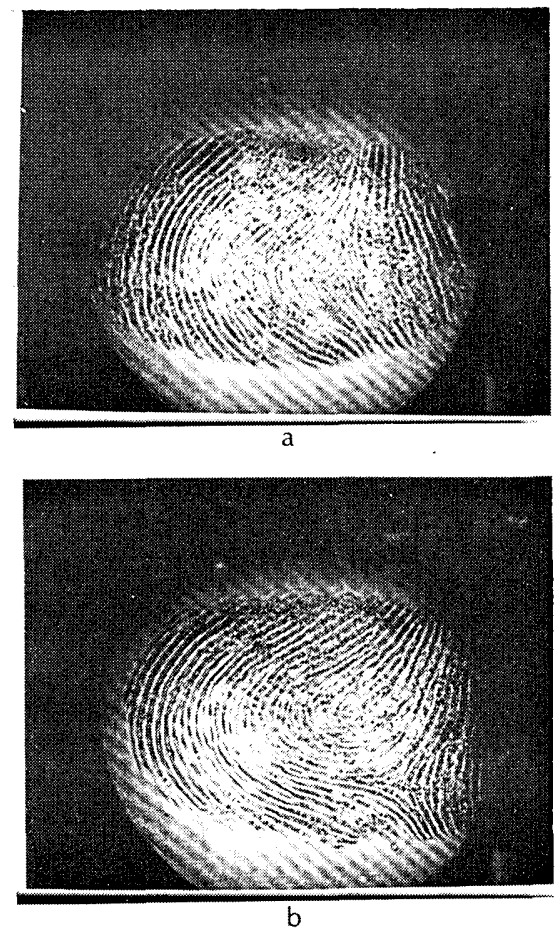

Fig. 5. Object fingerprints: (a) F2, (b) F3.

also be performed. In this case any output can be associated with a matched partial input.

\section{B. Experimental Apparatus}

The experimental setup is shown in Fig. 4. In the recording stage, a collimated beam (object beam) illuminates a transmissive object, a black-ink fingerprint on a slide. The object beam is transformed by a lens onto the Fourier plane where a holographic grating is formed by interfering with a collimated reference beam in dichromated gelatin (DCG). Two different objects, labeled F2 and F3 [Figs. 5(a) and (b)], were used in the experiment. F2 was interfered with a reference beam angle of $47^{\circ}$ at the Fourier plane, and F3 was interfered with a reference beam angle of $58^{\circ}$. 

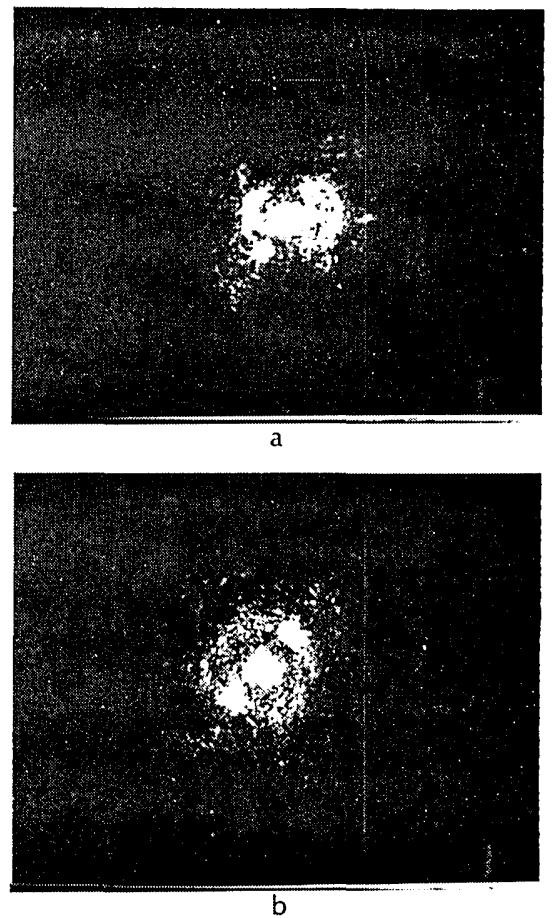

Fig. 6. Photograph of the Fourier transform plane for the object fingerprints: (a) Fourier transform plane for F2, (b) Fourier transform plane for fingerprint F3.

A considerable effort was made to optimize the DCG hologram. ${ }^{13}$ The system required optimal discrimination to permit a minimal partial-object input. The constraint of necessary switching power for the optically bistable interference filter dictated some minimum autocorrelation efficiency. These requirements led to a trade-off between system efficiency and discrimination. The object fingerprints had a fairly low modulation depth, and thus only $20 \%$ of the power was in the first order of the diffracted information ring of the Fourier transform [Figs. 6(a) and (b)]. In addition, the Fourier transform information had a large dynamic range-from 1:15 to 1:500 compared to the zero order. It was important to determine the appropriate reference beam intensity to give optimal grating visibility for the best efficiency while maintaining sufficient discrimination. It was also critical to ensure that the zero order saturated the DCG, so that the holographic fringes were suppressed. This eliminated the contribution to cross correlation that was due to the zero order. Even when the zero order saturates the DCG, some unwanted modulation occurs in the wings of the feature. Large diffraction from the zero order would destroy the discrimination because all objects have similar zero orders. It was found that an objectto-reference ratio of 20:1 in the Fourier transform plane (using the peak of the zero order as the object intensity) and $\sim 190-\mathrm{mJ} / \mathrm{cm}^{2}$ exposure gave $4 \%$ autocorrelation efficiency; this maximized the efficiencydiscrimination trade-off in this experiment.
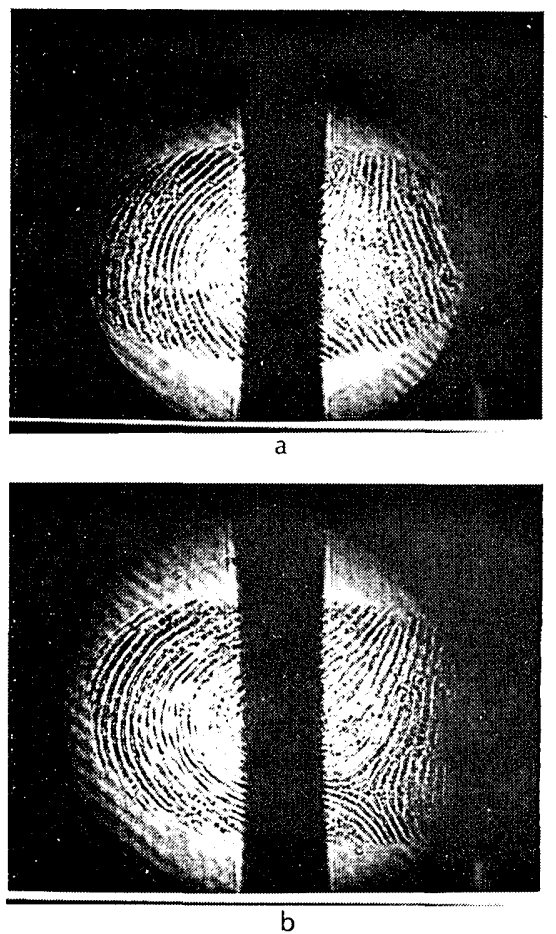

Fig. 7. Partially obstructed objects: (a) F2, (b) F3.

\section{Results}

When operating the system one of the objects was partially obstructed and illuminated [Figs. 7(a) and (b)], causing autocorrelation beams to be diffracted from the hologram at the two reference angles. The autocorrelation peaks can be seen for F2 and F3 in Figs. 8(a) and (b), respectively, for the unobstructed and partial cases. For the partial input case the discrimination ratios (autocorrelation intensity vs crosscorrelation intensity, in the correlation plane) of the correlation peaks were $50: 1$ with F2 as test object and 10:1 with F3. The reduced discrimination for partial F3 input was due to its smaller modulation depth which resulted in a larger zero-order content.

The autocorrelation peak for the partial-object input was of sufficient intensity $(\sim 3 \mathrm{~mW}$ in a $100-\mu \mathrm{m}$ diam spot) to switch the optically bistable interference filter into the transmissive mode (Fig. 9). The switching of the optically bistable interference filter into the transmissive state allowed the counterpropagating holding beam to read out the hologram. The autoassociatively reconstructed image due to a single readout beam [Fig. 10(a)] had a contrast ratio of 2:1 (the ratio of the switched holding beam transmission to the passive optically bistable interference filter transmission of the beam, or equivalently, the switching contrast of the optically bistable interference filter). The heteroassociative reconstruction yielded similar results, as seen in Fig. 10(b). Because the diffraction efficiency of the hologram was only $4 \%$ at least $75 \mathrm{~mW}$ of object input beam power was required, causing dynamic thermal damage in the DCG. The relatively 


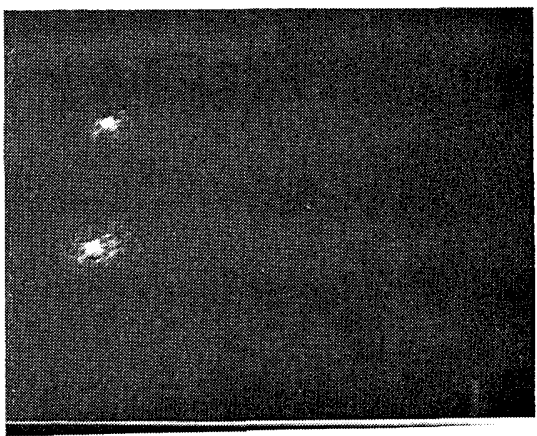

a

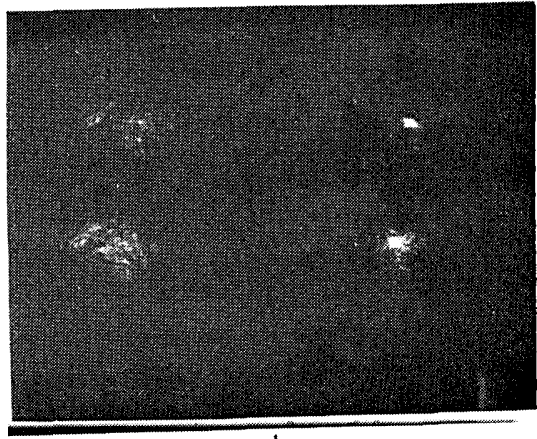

b

Fig. 8. Cross-correlation beams for partial and whole input objects. (a) Upper, partial F2 input; lower, whole F2 input. (b) Upper, partial F3 input; lower, whole F3 input.

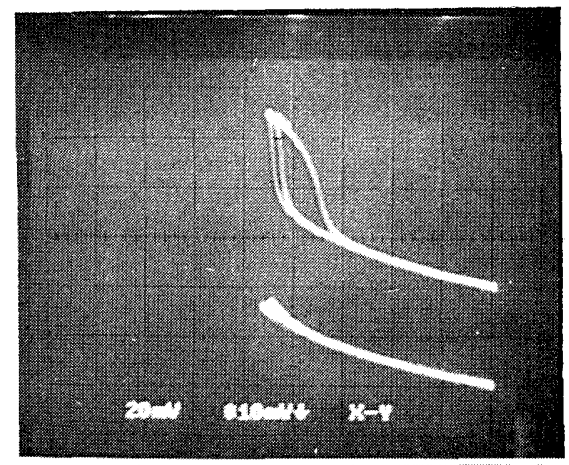

Fig. 9. Optically bistable interference filter response: Upper, switched on by the correlation input (vertically offset for clarity); lower, switched off. (Trace describes input holding beam intensity $-x$ vs transmitted holding beam intensity $y$ ).

poor quality of the reconstructed objects was due to this thermal damage in the DCG.

Decision-making was demonstrated on only one cross-correlation beam at a time, having determined that sufficient discrimination was present to preclude switching of both beams simultaneously. However, if two devices had been installed each would have produced an off-state recall (from the off-state linear transmission of the holding beam through the interference filter), further degrading the on-state recall discernibility.

In a previous experiment one object was stored, the letters $W X$, a partial input was recognized, and associative recall was executed (Fig. 11). In this case dis-

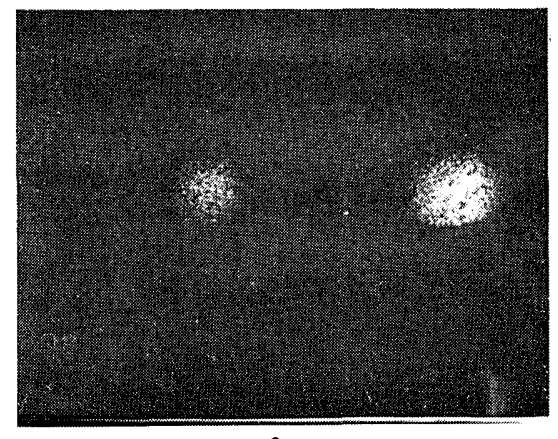

a

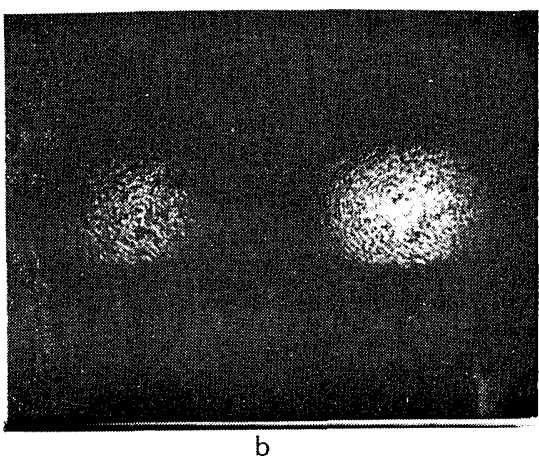

Fig. 10. Recalled object F2. (a) Autoassociatively recalled: left, switched off; right, switched on. (b) Heteroassociatively recalled: left, switched off; right, switched on.

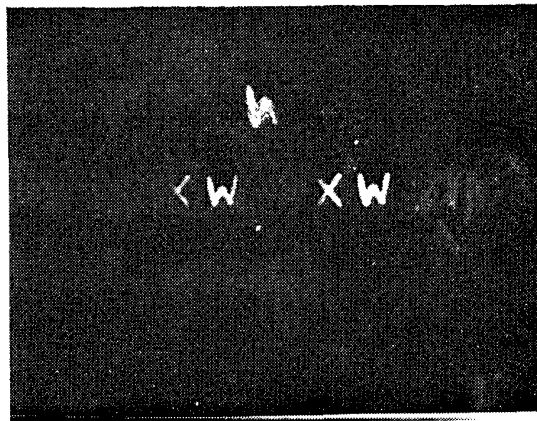

Fig. 11. Recognition of partial input for stored $W X$. Top, partial input; bottom left, recall switched off; bottom right, recall switched on.

crimination was not necessary (only one object was stored) so that increased cross-correlation efficiency could be obtained through the use of the zero-order. High quality recall was facilitated because thermal damage was not an issue.

\section{Discussion}

The two systems described used nonlinear devices to provide decision-making for optical processing. Although the spot-recognition system was more decision-element intensive, both systems made obvious the shortcomings of the interference filter devices (e.g., power requirements, uniformity). Although a substantial speed advantage can be gained by the use of GaAs etalon devices, they would certainly not alleviate the high power requirements of these systems. The 
speed advantage of the GaAs devices, however, may be the decisive factor in certain applications.

In associative memory the DCG holographic VanderLugt filter limited the performance of the system. Although the object employed was responsible for certain aspects of the experimental difficulty, even in ideal object conditions the diffraction efficiency achievable will be a limiting factor. This is aggravated as the number of stored objects is increased. ${ }^{14}$ Photorefractive materials are a possibility to increase the number of stored objects due to large material thickness, but typically the diffraction efficiencies are even more limited than with DCG holograms. The power throughput requirements dictated by subsequent nonlinear devices can also introduce serious complications due to the storage and erasure dynamics of photorefractive holograms. These dynamics can also introduce new flexibility to the holographic element.

\section{Conclusion}

Optical nonlinear devices were successfully employed as decision-making elements in two all-optical systems. However, the limiting aspects of the nonlinear devices and the holographic element were felt even in these comparatively simple systems.

Support from AFOSR, ARO, NSF, RADC/DARPA, SDIO, and the Optical Circuitry Cooperative is gratefully acknowledged. Special thanks to J. McCann for the phase gratings.

\section{References}

1. B. S. Wherrett, D. Hutchings, and D. Russell, "Optically Bi- stable Interference Filters: Optimization Considerations," J. Opt. Soc. Am. B 3, 351 (1986).

2. G. R. Olbright, N. Peyghambarian, H. M. Gibbs, H. A. Macleod, and F. Van Milligen, "Microsecond Room-Temperature Optical Bistability and Crosstalk Studies in ZnS and ZnSe Interference Filters with Visible Light and Milliwatt Powers," Appl. Phys. Lett. 45, 1031 (1984).

3. A. Huang, "Parallel Algorithms for Optical Digital Computers," in Proceedings, IEEE Tenth International Optical Computing Conference (1983), p. 13.

4. K.-H. Brenner, A. Huang, and N. Streibl, "Digital Optical Computing with Symbolic Substitutions," Appl. Opt. 25, 3054 (1986).

5. L. Wang et al., "Symbolic Substitution Using ZnS Interference Filters," Proc. Soc. Photo-Opt. Instrum. Eng. 752, 14 (1987).

6. J. J. Hopfield, "Neural Networks and Physical Systems with Emergent Collective Computational Abilities," Proc. Natl. Acad. Sci. U.S.A. 79, 2554 (1982).

7. T. Kohonen, Self-Organization and Associative Memory (Springer-Verlag, New York, 1984).

8. D. Psaltis and N. Farhat, "Optical Information Processing Based on an Associative-Memory Model of Neural Nets with Thresholding and Feedback," Opt. Lett. 10, 98 (1985).

9. Y.S. Abu-Mostafa and D. Psaltis, "Optical Neural Computers," Sci. Am. 256(3), 88 (1987).

10. E. G. Paek and D. Psaltis, "Optical Associative Memory Using Fourier Transform Holograms," Opt. Eng. 26, 428 (1987).

11. D. Anderson and M. C. Erie, "Resonator Memories and Optical Novelty Filters," Opt. Eng. 26, 434 (1987).

12. Y. Owechko, G. J. Dunning, E. Marom, and B. H. Soffer, "Holographic Associative Memory with Nonlinearities in the Correlation Domain," Appl. Opt. 26, 1900 (1987).

13. T. G. Georgekutty and H.-K. Liu, "Simplified Dichromated Gelatin Hologram Recording Process," Appl. Opt. 26, 372 (1987).

14. J. Hong and D. Psaltis, "Storage Capacity of Holographic Associative Memories,” Opt. Lett. 11, 812 (1986).

\author{
LASER OPTICS \\ The ability to control a laser output for effective \\ use requires the practical application of optics in a \\ highly specialized area where unusual optical phenomena \\ occur. Applies the principles of geometric \& wave \\ optics to the specific components used for developing \\ and controlling laser outputs. Workshops and problem \\ sessions are included to allow the student to apply \\ these principles to simulated optical problems (phenomena \\ occurring with lasers). Also included will be an introduction \\ to matrix optics \& its application to simple optical \\ systems. \\ ENGINEERING TECHNOLOGY INSTITUTE \\ P.O. Box 8859 \\ Waco, TX 76714-8859 \\ (817) 772-0082 \\ 1-800-367-4238 \\ 5 Days \\ Cost: $\$ 910.00$ \\ August 1-5, 1988 \\ Washington D.C.
}

